\title{
Computer Aided Diagnosis Based on Artificial Intelligence in Providing Support for Health Services in Rural Areas
}

\author{
Retno Supriyanti ${ }^{1}$ \\ ${ }^{1}$ Electrical Engineering Dept, Jenderal Soedirman University
}

\begin{abstract}
The health service system in Indonesia is still a homework that needs to be addressed by the government. Both in terms of equity, regulation, and integration between offline and online systems. As for what is happening now, there are at least some obstacles that need to be addressed and addressed as soon as possible. On the other hand, health development should beseen as an investment to improve the quality of human resources and support economic development, and have an essential role in poverty reduction efforts. Today's primary health development problems include the high disparity in health status between socioeconomic levels, regions, and urban and rural areas. In general, the health status of people with high socioeconomic levels in western Indonesia and urban areas tends to be better. On the other hand, the health status of people with low socioeconomic status in eastern Indonesia and rural areas is still lagging. The quality, equity, and affordability of health services are also still low. The quality of service is an obstacle because medical personnel is minimal and equipment is inadequate. In terms of numbers, the ratio of health workers to the population to be served is still low. The affordability of services is closely related to the number and distribution of healthfacilities. In terms of the limitations of medical equipment, this is due to the high price of the medical equipment. Besides, special training is also needed in medical equipment, which training takes quite a long time, and the material is quite complex. With these conditions, it is necessary to optimize technology to develop medical equipment, especially diagnostic supportequipment that is cheap, easy to use, and has high accuracy. Our research aims to address optimizing image processing technology and artificial intelligence in developing technology to support health services in rural areas. We tried to implement several cases of health services that often occur in rural areas, namely early detection of cataracts, optimizing the use of ultrasound machines, optimizing the use of low- quality MRI machines in the diagnosis of Alzheimer's, using microscopic images to identify leukocytes and using X-ray images to support the diagnosis. COVID19. Of the several modulesthat we have developed for health care support technology, the results show promisingprospects with a good level of accuracy, ease to use, and use modalities that already exist in the lowest health service units to reduce prices. It is hoped that this image processing and artificial intelligence-based health service support module can be used in rural areas to overcome disparities in health services in Indonesia.
\end{abstract}

Keywords : limited health services, Easy-to-use, High accuracy, Low-cost, Rural areas 